\title{
Cerebral microcirculation during severe head injury after a contusion and acceleration experimental model in sheep
}

\author{
Judith Bellapart ${ }^{1}$, Kylie Cuthbertson ${ }^{2}$, Kimble Dunster ${ }^{3}$, Sara Diab ${ }^{3}$, David G. Platts ${ }^{4}$, \\ Christopher Raffel ${ }^{4}$, Levon Gabrielian ${ }^{5}$, Adrian G Barnett ${ }^{6}$, Jennifer Paratz ${ }^{1}$, Rob Boots ${ }^{1}$, \\ John F. Fraser ${ }^{7,8}$
}

${ }^{1}$ Corresponding Author: Dr Judith Bellapart-Rubio. MD, ESICM, CICM. Critical Care Research Group, University of Queensland; Intensive Care Department, Royal Brisbane and Women's Hospital, Butterfield Street, Herston, 4025, QLD, Australia. FAX: +61736363542, Phone +61736368897, email: judithbellapart@gmail.com

${ }^{1}$ Jennifer Paratz: School of Medicine, University of Queensland, Herston, 4025, QLD and Griffith University, Parkland Drive, Southport 4215, Australia. FAX: +61736363542, Phone+61736368897, email: j.paratz@uq.edu.au

${ }^{1}$ Rob Boots: Intensive Care Department, Royal Brisbane and Women's Hospital, Butterfield Street, Herston, 4025, QLD, Australia. FAX: +61736363542, Phone+61736368897, email: r.boots@uq.edu.au

${ }^{2}$ Kylie Cuthbertson: Histopathology Department, Royal Brisbane and Women's Hospital, Herston, 4025, QLD, Australia. FAX: +61736468908, Phone: +6136461797 email: Kylie.Cuthbertson@health.qld.gov.au.

${ }^{3}$ Kimble Dunster: Critical Care Research Group, University of Queensland; Medical Engineering Research Facility, Queensland University of Technology, Stafford Heights 4053, QLD, Australia. FAX: +61731386937, Phone +61731386938, email: k.dunster@ qut.edu.au

${ }^{3}$ Sara Diab: Critical Care Research Group, University of Queensland; Medical Engineering Research Facility, Queensland University of

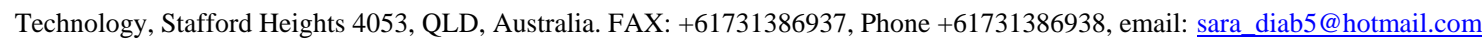

${ }^{4}$ David G. Platts: Critical Care Research Group, University of Queensland; Department of Cardiology, The Prince Charles Hospital, Chermside 4032, QLD, Australia. FAX: +61731394388, Phone: +61731394000, email: dgplatts@ hotmail.com

${ }^{4}$ Christopher Raffel: Critical Care Research Group, University of Queensland; Department of Cardiology, The Prince Charles Hospital, Rode road, Chermside 4032, QLD, Australia. FAX: +61731394388, Phone: +61731394000, email: ocraffel@gmail.com

${ }^{5}$ Levon Gabrielian: Medical School Research Centre, Frome road, Adelaide, 5005 SA Australia. FAX: + 61883135573, Phone: +61883135571, email: levon.gabrielian@gmail.com

${ }^{6}$ Adrian Barnett: Institute of Health and Biomedical Innovation \& School of Public Health and Social Work, Queensland University of Technology, 60 Musk Avenue, Kelvin Grove, 4059 QLD, Australia. FAX: +61731382006, Phone +6131382000, email: a.barnett@qut.edu.au

${ }^{7,8}$ John F. Fraser: Critical Care Research Group, University of Queensland; Intensive Care Department, The Prince Charles Hospital, Rode road, Chermside 4032, QLD, Australia. FAX: +61731394388, Phone: +61731394000, email: fraserjohn001@gmail.com

Keywords: Brain trauma, cerebral blood flow, axonal damage 


\section{Abstract}

Background: Cerebral microcirculation after severe head injury is heterogeneously distributed and temporally variable. Microcirculation changes have been found in association to the degree of tissue injury although there are currently no studies demonstrating how histological damage relates to cerebral regional blood flow at specific anatomical regions.

Objective: To test the hypothesis that cerebral microcirculation after severe head injury, is compromised specifically at the anatomical regions of highest tissue disruption and that these changes are time dependant even from the first hours after injury.

Methods: A sample of eight merino sheep exposed to a contusion and accelerationdeceleration brain injury. Cerebral microcirculation was directly quantified using ultrasound guided injection of colour coded microspheres into the left cardiac atrium to ensure systemic and homogeneous distribution. Histological analysis used amyloid precursor protein staining to identify early areas of axonal injury in predefined anatomical regions. A mixed effects regression model assessed the hourly blood flow results during four hours after injury.

Results: Regional microcirculation blood flow values for each subject and tissue region over the entire study time, including baseline, ranged between $20 \mathrm{ml} / 100 \mathrm{~g} / \mathrm{min}$ and $80 \mathrm{ml} / 100 \mathrm{~g} / \mathrm{min}$. RMBF values for each subject and tissue region over the four hours from baseline were reduced in all subjects and all regions at all times, but the mean confidence intervals crossed the horizontal ratio of 1 indicating that such reduction was not statistically significant.

Conclusion: After severe head injury, cerebral microcirculation at the ipsilateral and contralateral site of a contusion in addition to the ipsilateral thalamus and medulla shows a consistent decline over the first four hours after injury, when compared with baseline. Although not statistically significant, a reduction in cerebral microcirculation and an 
expression of APP staining as indicator for axonal damage was found to be related to the severity of head injury. 


\section{Introduction}

Severe head injury is commonly the result of a combination of contusion with accelerationdeceleration forces leading to cellular breakdown, cytogenic and vasogenic edema, impaired cerebral autoregulation and perfusion mismatch [1]. Irreversible cellular damage has been described specifically in areas where microcirculation is critically reduced $[2,3]$ and in areas where critically low levels of Partial Pressure of Tissue Oxygenation (PTi02) have been maintained (REF) despite the controversies on the impact of anaemia upon cellular oxygenation and tissue injury $[4,5]$. Whilst the assessment of cerebral regional microcirculation is still largely experimental, its role in comprehending the pathophysiology of severe head injury to provide insights into clinical management is essential. However, management of head injury patients is commonly based on systemic measures that ensure global perfusion and oxygenation parameters, without specifically targeting cerebral metabolic demands, cerebral tissue oximetry or regional distribution of blood flow. So, whilst multi-monitoring modalities are considered to be the benchmark practice within neuro-critical units [6] and several studies advocate for the use of PTi02 as a surrogate for neuronal recovery (REFS from anaemia review), the significance of clinical strategies aiming to increase the PTi02 levels is still unclear. Despite the previously demonstrated relationship between regional microcirculatory blood flow (RMBF), tissue metabolic demands [7] and cerebral hypoperfusion leading to irreversible cellular damage, there are no studies quantifying the temporal variability of RMBF in different cerebral anatomical regions after severe head injury or its close relation to the degree of axonal damage and the state of cerebral tissue oxygenation.

We hypothesised that after severe head injury cerebral microcirculation heterogeneity may correlate with the severity of the injury with maximal RMBF reduction seen within the areas of severe tissue disruption. 
This study aims to directly quantify the temporal changes in ovine RMBF at specific cerebral regions, particularly within the areas of maximal axonal damage and the area corresponding to the ischemic penumbrae when compared to contralateral and non-injured regions.

\section{Materials and methods}

\section{Animal care and preparation}

Experimental procedures were approved by the Animal Ethics Committee of the Queensland University of Technology. Sheep were used because of their cerebral anatomical similarities with humans, specifically the cerebral gyrencephalic surface allowing better examination of the grey-white matter; a well-defined physiology of the ovine haemoglobin dissociation curve [8] and extensive experimental neuroscience experience using this animal model [911]. A convenience sample of eight Merino weathers weighing $40 \pm 5 \mathrm{~kg}$ were instrumented with a triple lumen central line (Cook Medical. Queensland, Australia) and two 16Fr introducer sheaths in the right internal jugular (RIJ) vein. Via the central line, general anaesthesia was given using ketamine with an initial bolus of $5 \mathrm{mg} / \mathrm{kg}$ and maintenance infusion between $0.5-1 \mathrm{mg} / \mathrm{kg} / \mathrm{h}$. Sedation was achieved with a combined infusion of midazolam $(0.5 \mathrm{mg} / \mathrm{kg} / \mathrm{h})$, fentanyl $(10 \mathrm{mcg} / \mathrm{kg} / \mathrm{h})$ and alfaxalon $(6 \mathrm{mg} / \mathrm{kg} / \mathrm{h})$. This anaesthetic combination previously used in a mild head injury study (REF 2 of 5) demonstrated cardiovascular stability without alteration in cerebral microcirculation in sheep [12]. Hydration was maintained with an infusion of Hartmann's solution up to a rate of 2 $\mathrm{mL} / \mathrm{kg} / \mathrm{h}$, titrated to maintain a central venous pressure (CVP) of 6-10 mmHg. Cardiovascular monitoring included cardiac output and vascular resistances via a Swan-Ganz catheter as previously described [13] (and 2 of 5 study) and a 5F umbilical vessel catheter (Argyle, Tyco HealthCare, Mansfield, MA, US), placed in the right femoral artery to allow a withdrawal of blood at a rate of $10 \mathrm{ml} / \mathrm{min}$. Orotracheal intubation used a size $10 \mathrm{~mm}$ endotracheal tube 
(SIMS Portex, UK). Sheep were ventilated at 12 breaths per minute with tidal volumes of 8 $\mathrm{mL} / \mathrm{kg}$ and $5 \mathrm{~cm} \mathrm{H}_{2} \mathrm{O}$ of PEEP with an initial $\mathrm{FiO} 2$ of 1.0 with the $\mathrm{FiO} 2$ and respiratory rate titrated to maintain a partial pressure of oxygen $\left(\mathrm{PaO}_{2}\right)$ of $>95 \mathrm{mmHg}$ and normocapnia. PEEP levels were maintained at $5 \mathrm{~cm} \mathrm{H}_{2} \mathrm{O}$ to minimise de-recruitment consistent with common clinical practice and known to have no effect on cerebral blood flow [14] (and ref 2 of 5). Neuro-monitoring included a Lycox PTi02 probe and an intracranial Pressure (ICP) monitor, (Oxford Optronics, Ltd, Oxford, United Kingdom). Craniectomies were performed prior to injury but dura was left intact to avoid any effect on intracranial pressure. Craniotomies for the insertion of both probes were performed exactly $15 \mathrm{~mm}$ lateral to the sagittal suture and anterior to the coronal suture $[11,15]$. Probes were introduced at $35 \mathrm{~mm}$ and $15 \mathrm{~mm}$ from the skull respectively after piercing the dura with the end of the tip located at the white matter as previously performed [16].

In order to avoid red blood cell storage into the sheep's spleen and maintain stable haemoglobin throughout the study $[17,18]$, ligation of the splenic artery was performed as in previous studies [16] (Refs 1 of 5 and 2 of 5 ).

The monitoring and preparation phase was completed with an intracardiac echocardiography (ICE) guided insertion of a transeptal catheter into the left cardiac atrium (LA). Echocardiography images were obtained using an Acuson Sequoia C512 scanner (Siemens, California). Transeptal puncture and insertion of a pigtail catheter into the LA followed previously described methods [19].

\section{Trauma model}

Under anaesthesia, a blunt injury was applied over the left temporal bone using a nonpenetrating stunner (model MKL, Karl Schermer, Ettlingen Germany) with the intention to 
generate a severe head injury but without leading to brain death, causing both contusional and acceleration injury [20]. After injury, burr holes were formalised and the dura pierced for the insertion of pressure and tissue-oximetry probes as previously described (Ref 2 of 5). The main difference from previous study (Ref 2 of 5), was that in this study the goal was to achieve a severe head injury. This was achieved by recruiting animals of significantly lower weight, (40-45 kg compared to $65-70 \mathrm{~kg}$ ). The impact force used remained unchanged expecting a greater injury in a smaller brain.

\section{Protocol for microspheres injection}

At each time point (T0 corresponding to baseline, T1-to-T4 corresponding to first to fourth hours after trauma respectively) an injection of colour-coded microspheres (E-Z TRAC; Interactive Medical Technology, Los Angeles, CA) was done through the LA pigtail catheter as performed previously [16, 21] (2 of 5). Randomly assigned colours at each time-point and subject minimised selection biases and allowed the tracking of RMBF at specific anatomical regions for each time point and subject. Five different colours (purple low, purple high, pink high, yellow high and coral low) were recommended by the manufacturer $\underline{\text { www.microspheres.net }}$ to facilitate cytometric counts. Each injection included a homogeneous mixture of one colour-microsphere with a density of 5 million spheres in 0.8 $\mathrm{ml}$. This microsphere density has been used [22] without causing microvascular occlusion. Microspheres were injected thirty seconds after the initiation of the withdrawal pump. The withdrawal pump was connected to the arterial catheter with the intention to withdraw blood at a rate of $10 \mathrm{ml} / \mathrm{min}$ to obtain the reference blood sample required for the calculation of tissue RMBF. Two minutes after commencement of the withdrawal pump the reference blood sample collection was completed and the inline catheter was flushed with Tween 80 reagent to recover microspheres entrapped in the line [23]. 
This protocol otherwise reproduced that of previously published studies [16] (2 of 5).

\section{Euthanasia and post-mortem tissue manipulation}

After five hours of continuous monitoring and microsphere injection, sheep were euthanized under non-recovered anaesthesia with a bolus injection of $0.5 \mathrm{~mL} / \mathrm{kg}$ of sodium pentobarbitone. After confirmation of death (asystole arrest), the brain was extracted, weighed and fixed with $10 \%$ formalin for three weeks.

\section{Brain harvesting technique}

Brain harvesting was facilitated with the use of a round reciprocating saw sectioning approximately $5 \mathrm{~cm}$ bone sections from the temporal region to the frontal sinuses. These bone sections were removed with simultaneous dissection of the dura to avoid parenchymal tearing. Once the brain was fully exposed, the olfactory bulbs, optic chiasm, tentorium and cranial nerves were progressively sectioned as the brain was lifted from the base of the skull. This approach achieved a controlled dissection avoiding injury to the brain tissue [12]. Brains were weighted prior to $10 \%$ formalin fixation for a minimum of three weeks.

\section{Tissue sampling model}

After the period of immersion fixation, brains were macroscopically inspected to assess for cortical impacts, haemorrhages or the presence of a contra-coup injury. Following external inspection, each brain was sectioned creating $5 \mathrm{~mm}$ antero-posterior slices. Each slice was macroscopically inspected to identify regions of maximal contusion. Cone samples were extracted from pre-defined anatomical regions these labelled as follows: AL corresponding to the core of contusion at the side of the injury; BL, the peri-contusional region at the side of the injury; $\mathrm{AR}$, the mirror region to the core of contusion on the contralateral side; $\mathrm{BR}$, the 
mirror region to the peri-contusional region on the contralateral side; $\mathrm{C}$, the thalamus at the ipsilateral side to the contusion and D corresponded to the medulla, this method was reproduced from a previous study (Ref 1 and 2 of 5). Adjacent tissue blocks were assigned for both cytometric and histological analysis, to superimpose histology with cerebral blood flow data.

Samples from skin, kidney, heart and spleen were extracted from each sheep to demonstrate systemic distribution of microspheres as well as to confirm the presence of splenic infarcts representing successful spleen ligation respectively.

\section{Quantification of microvascular blood flow}

The total amount of each colour microsphere imbedded in each particular region of interest used a previously validated cytometric analysis (Ref 24). RMBF was calculated from the known microsphere concentration injected into the arterial supply and the amount of each colour microspheres found in each reference blood sample. [24]. RMBF represents the proportion of microspheres trapped in the targeted tissue in relation to the total quantity of spheres per $\mathrm{mL}$ of blood per minute of the reference sample using the equation:

$\operatorname{RMBF}(\mathrm{mL} / \mathrm{min} / \mathrm{g})=($ Total tissue spheres $) /[($ Tissue weight, $\mathrm{g}) \times($ Reference Spheres $/ \mathrm{mL} / \mathrm{min})]$ [25]

Cytometric analysis was performed at the Interactive Medical Technology (IMT), Los Angeles, California, US www.microspheres.net.

\section{Immunohistochemestry processing}

Immunohistochemistry analysis was performed at the neuro-pathology laboratory, Royal Brisbane and Women's Hospital, QLD, Australia. Immunohistochemistry used a Leica Novolink Polymer Detection Systems Kit (Leica Microsystems Pty Ltd, North Ryde, 2113 Australia) as per manufacturer's instructions www.leica-microsystems.com. Sections had paraffin removed through a series of xylene immersions and re-hydrations. Antigen retrieval 
was carried out using Leica BOND ER1 solution. Sections were incubated with a protein block. The primary antiserum made up in Leica BOND Antibody Diluent was applied to the sections.

\section{Immunohistochemestry and Hematoxillin-Eosin scoring and Interpretation}

Immunohistochemistry analysis using APP antibodies staining was applied to all targeted areas of interest. APP antibody staining was used to identify areas of tissue with high density of APP staining, specifically at regions of interest. APP expression is considered to be a very early marker of neuronal damage [26] and therefore suitable as an early histopathological marker for a 4 hour study. A pre-existing grading system measuring the presence of APP and structured into 3 qualitative categories dependent upon the severity of injury was applied. This qualitative score defined the APP staining as: Mild: a focal contusion with APP labelling limited to the site of injury, Moderate-: a pattern of APP staining greater than one hemisphere, greater than half a hemisphere or less than half a hemisphere and Severe: characterised for the presence of diffuse staining and sub-classified as either diffuse vascular injury, diffuse axonal injury with macroscopic haemorrhage, diffuse axonal injury with microscopic haemorrhage/tissue tears or diffuse axonal injury [12]. Each animal had samples for both cytometric count of RMBF and immunohistochemistry at each anatomical region of interest with the intention to superimpose flow data with histopathology data at each area of interest and at each time point before and after severe head injury.

\section{Statistical Analysis}

RMBF raw data for each sheep was plotted over time and time averaged for the study cohort. The ratio of RMBF from 1 to 4 hours after injury (T1 - to - T4) compared to baseline (time 
zero - T0) was also plotted, with a ratio below one representing a decrease in RMBF over time and a ratio over one representing an increase in RMBF over time.

To test for statistical differences we used a mixed effects regression model of the ratios from times T1 to T4 with a random intercept for each sheep to control for correlated data from the same sheep. We fitted an independent effect at each time (T1 to T4) as we were uncertain of how the change in RMBF over time would look. All the plots and regression models were run separately for each area studied (AR, BR, AL, BL, C and D). We used the R software version 3.1.2 for all analyses (www.r-project.org).

\section{Results}

A convenience sample of eight sheep weighting 40 - to - $45 \mathrm{~kg}$ was used. Subjects remained cardio-vascularly stable throughout the entire study time even after a severe head injury (Table I), except for one subject (subject number 5) who became profoundly vasoplegic after injury in association with bilateral fixed mydriasis and a comminuted skull fracture. These signs suggested high intracranial pressures and a cerebral herniation. Insertion of intracranial pressure probes was not feasible; therefore ICP could not be quantified due to the fractures. Systemic variables known to influence cerebral perfusion and reflecting peripheral oxygen extraction, such as cardiac output (CO) and Central Venous Oxygen Saturation (SVc02) were stable throughout the study (Table II). $\mathrm{PaO} 2$ and partial pressure of arterial $\mathrm{CO} 2(\mathrm{PaCO} 2)$ affecting oxygen delivery to tissues and cerebral blood volume, were unchanged during the study period (Table III). Blood hemoglobin concentration was maintained through the study via a spleen ligation, to limit effects on oxygen delivery to tissues and microcirculation rheology (Table IV). Cerebral Partial Tissue Oxygenation (PTi02) was recorded in every subject from hour one after trauma as PTi02 probes were inserted after formalising the craniectomies (Table V). PTi02 amongst subjects showed compromised levels of tissue 
oxygenation through all times except for subject number three which maintain normal and hyperaemic values. PTi02 was reflecting only tissue oxygenation at the ipsilateral side to the injury.

RMBF Analysis

RMBF values for each subject and tissue region over the entire study time for each subject and tissue region are shown in Figure 1 with their means in Figure 2. RMBF ratios for each subject and tissue region over the four hours from baseline are shown in Figure 3. The horizontal line at a ratio of 1 indicates no changes on RMBF from baseline. RMBF mean ratios from all subjects per anatomical region and time are in Figure 4. RMBF means and 95\% confidence intervals represented by the vertical lines are shown in Figure 5, with statistical significance indicated by those confidence intervals that do not cross the horizontal reference line of 1 (no change from baseline). RMBF for all anatomical regions and at all times were reduced from baseline (pre-injury) but were not significantly different from baseline.

Regional flow in the y-axis is represented as per 1mg tissue weight, showing that in our study, physiological RMBF values were found, when normalised to a $100 \mathrm{mg}$ tissue weight.

\section{APP Scoring}

Results for APP scoring are summarised in Table VI. Minimal APP staining pattern was seen predominantly in all anatomical regions with moderate and severe APP staining pattern also present and distributed homogeneously over all anatomical regions. 


\section{Extra-cranial tissues}

Direct quantification of RMBF was also performed at extra-cranial regions, in particular, at the skin, heart, kidney and spleen (Figure 6). The aim was to demonstrate systemic distribution of colour coded microspheres as a proof of concept during all times. In addition, minimal presence of RMBF at the spleen aimed to demonstrate that spleen artery ligation had been performed efficiently.

\section{Discussion}

This study extends our understanding of the effect of increased traumatic brain injury on the micro-circulation. Severe head injury as opposed as mild (REF 2 of 5) was induced with the use of smaller animals, the appliance of the same stunner force and the maintenance of all other methodological aspects of the study, generating two populations of subjects, statistically comparable.

The most important finding on this severe head injury study is the presence of a reduction of RMBF from baseline (RMBF pre-injury) in all subjects, at all anatomical regions of interest and at all times through the study length, as seen in figure 4. This finding is consistent with the a priori expectations regarding cerebral microcirculation after severe head injury; hence, contrasts with the presence of heterogeneity and temporal variability found in a mild head injury study (REF 2 of 5), as a consistent reduction on cerebral flow is found in all targeted regions during the four hours of study, although this reduction in RMBF is not statistically significant (Figure 5), likely to having used a reduced animal cohort; however these experimental studies focus their relevance in their design and plausibility of findings.

When cerebral RMBF values were normalised to a global unit of measure ( $\mathrm{ml} / 100 \mathrm{~g}$ tissue /min), physiological range of RMBF values were found through all anatomical regions. 
Furthermore, mean cerebral RMBF values at baseline were above the ischemic threshold of $15-20 \mathrm{ml} / 100 \mathrm{~g} / \mathrm{min}$ [27] despite the presence of severe head injury. This is suggesting that in this severe head injury model, RMBF although consistently reduced from baseline, was maintained within normal ranges. This finding was not expected as the induced trauma was sufficient to generate clinical complications such as compound skull fractures, traumatic mydriasis and significant acceleration deceleration drift of the head seen in some of our subjects. However, it is compatible with the APP staining results which demonstrated a predominant mild scoring, suggesting the presence of mild axonal injury, instead of severe. RMBF data in this severe head injury study was compared with the previous study corresponding to mild head injury. No statistical significance was found within RMBF distribution and quantification throughout all the anatomical regions of interest and during the four hours of study within these two cohorts.

The cerebral RMBF decline from baseline in this study was not related to an increase in ICP nor a reduction in CPP as these parameters were stable and in normal ranges for all subjects except for subjects numbered six to eight (Table I). This suggests that reductions in cerebral RMBF post injury may be also related to early inflammatory changes and tissue disruption after trauma even prior to the establishment of high intracranial pressure. This finding raises concerns related to cerebral vulnerability to ischemia even in the absence of poor intracranial compliance. This study's implications and translation into clinical practice is limited by the timeframe of the study, as it focuses on cerebral microcirculation within four hours after injury. However, as previously emphasized, the novelty of this study design relies on the microcirculatory quantification using cytometric methods when targeting specific anatomical regions, confirming the reduction of RMBF from the very first hour after trauma. Clinical implications are related to the risk of developing cerebral infarcts even with the co-existence 
of a preserved ICP suggesting that efforts should be oriented to optimise cerebral perfusion, even within the first four hours after head injury, regardless the level of ICP.

Histopathological analysis using APP staining on the adjacent anatomical regions where flow cytometric count had been performed showed predominantly minimal APP staining corroborating the presence of minor degree of axonal injury. Axonal disruption can be histologically graded by quantification of APP staining with a good correlation between the amount of staining and the intensity of axonal damage [28-31]. However, even in the setting of mild axonal injury, a time-dependent reduction in cerebral RMBF close to ischemic thresholds was found in all regions, from baseline. This is an important finding as it may indicate that even in situations where axonal damage is not derived from the primary injury; there is still the potential for the development of cerebral infarcts. Whilst this theoretical principle is widely accepted in neurocritical care, this study compares and contrasts for first time the interrelation between tissue damage and microcirculation in experimental models at specific anatomical regions, using histopathology and quantification of microcirculation analysed concurrently.

From the APP validated scoring we can imply that even in this severe head injury model, APP staining suggests that the cerebral injury can be mild or moderate (Table VI). However, whilst mild staining was distributed in the totality of the supratentorial regions, with only moderate to severe axonal injury at the medulla regions in the setting of mild injury; after a more severe injury, an heterogeneous distribution of axonal injury including mild to severe APP staining pattern, was found at both supratentorial and medullar regions. APP staining still remains an experimental marker in the field of neurotrauma, but it is reproducible and its applicability has been validated, used to define the distribution of axonal damage, as demonstrated in this study. 
Cytometric analysis in other organs, in particular the skin and spleen are shown in figure 6. RMBF in spleen showed negligible perfusion as consistent with spleen ligation. Spleen ligation allowed the maintenance of a steady-state level of haemoglobin, particularly during a stress response phase when splenic red blood cell storage is well described in ovine models [18]. Splenic RMBF in spleen was expressed in $\mathrm{ml} / \mathrm{g} / \mathrm{min}$, varying between $0.0030-0.0036$ $\mathrm{ml} / \mathrm{g} / \mathrm{min}$, a thousand fold less than normal controls [13]. This finding confirms that our splenic artery ligation was successful as well proven by the necrotic aspect of the spleen after harvesting. The main effect derived from the arterial spleen ligation is found on the stability of haemoglobin in all subjects and over all times after injury (Table IV); suggesting that the temporal changes on cerebral $\mathrm{RMBF}$ and $\mathrm{PTiO} 2$ seen in our subjects were not related to anaemia. This is an important finding as this reflects the state of cerebral microcirculation after trauma in conditions of baseline haemoglobin, a significant confounder in microcirculation. Although clinical extrapolations are not possible considering the experimental nature of this study, it is important to highlight that the haemoglobin levels in this cohort of subjects are to a degree higher to those currently accepted within clinical practice, especially amongst physicians tolerating a restrictive transfusion threshold.

In this study, PTi02 values in five of the eight subjects studied, maintained normal levels of tissue oxygenation, despite the presence of a decay in RMBF through time from baseline. One patient (subject five) had no recordable PTi02 as probes could not be safely introduced due to the presence of compound cranial fracture. These findings indicate that despite the global reduction in $\mathrm{RMBF}$ from baseline, the maintenance of above-ischemic perfusion thresholds may be sufficient to preserve tissue oxygenation, emphasizing the relevance of preserving cerebral perfusion even from early hours post trauma. Of note, RMBF was not related to the state of $\mathrm{CPP}$ but rather the preservation of cerebral microcirculation. 
It is difficult to derive clinical correlates in humans from experimental models. The study was of much shorter length then the clinical course of a human head injury. Although longer times of monitoring could have demonstrated a wider view of physiopathology processes affecting microcirculation after trauma, the focus of this study was the early changes in RMBF in specific regions of interest and in combination with the early expression of APP as a reliable marker of axonal damage.

\section{Conclusion}

After severe head injury, cerebral microcirculation at the ipsilateral and contralateral site of a contusion in addition to the ipsilateral thalamus and medulla shows a consistent decline over the first four hours after injury. Although non statistically significant, a reduction in cerebral microcirculation and an over-expression of APP staining as indicator for axonal damage are related to the degree of head injury.

\section{Acknowledgements}

The authors thank the Royal Brisbane and Women Research Foundation for their funding contribution

\section{Declaration of Interests}

The authors report no conflicts of interest.

\section{REFERENCES}

1. Werner C, Engelhard K: Pathophysiology of traumatic brain injury. Br J Anaesth 2007, 99(1):4-9.

2. Menon DK, Coles JP, Gupta AK, Fryer TD, Smielewski P, Chatfield DA, Aigbirhio F, Skepper JN, Minhas PS, Hutchinson PJ et al: Diffusion limited oxygen delivery following head injury. Crit Care Med 2004, 32(6):1384-1390.

3. Schwarzmaier SM, Kim SW, Trabold R, Plesnila N: Temporal profile of thrombogenesis in the cerebral microcirculation after traumatic brain injury in mice. J Neurotrauma, 27(1):121-130. 
4. Bellapart J, Boots R, Fraser J: Physiopathology of anemia and transfusion thresholds in isolated head injury. J Trauma Acute Care Surg, 73(4):997-1005.

5. Bratton SL, Chestnut RM, Ghajar J, McConnell Hammond FF, Harris OA, Hartl R, Manley GT, Nemecek A, Newell DW, Rosenthal G et al: Guidelines for the management of severe traumatic brain injury. XV. Steroids. J Neurotrauma 2007, 24 Suppl 1:S91-95.

6. Bhatia A, Gupta AK: Neuromonitoring in the intensive care unit. II. Cerebral oxygenation monitoring and microdialysis. Intensive Care Med 2007, 33(8):13221328 .

7. Kelly DF, Kordestani RK, Martin NA, Nguyen T, Hovda DA, Bergsneider M, McArthur DL, Becker DP: Hyperemia following traumatic brain injury: relationship to intracranial hypertension and outcome. J Neurosurg 1996, 85(5):762-771.

8. Samaja M, Gattinoni L: Oxygen affinity in the blood of sheep. Respir Physiol 1978, 34(3):385-392.

9. Cernak I: Animal models of head trauma. NeuroRx 2005, 2(3):410-422.

10. Ommaya AK, Gennarelli TA: Cerebral concussion and traumatic unconsciousness. Correlation of experimental and clinical observations of blunt head injuries. Brain 1974, 97(4):633-654.

11. Van Den Heuvel C, Lewis S, Wong M, Manavis J, Finnie J, Blumbergs P, Jones N, Reilly P: Diffuse neuronal perikaryon amyloid precursor protein immunoreactivity in a focal head impact model. Acta Neurochir Suppl 1998, 71:209-211.

12. Bellapart J, Cuthbertson K, Skerman J, dunster KR, Diab S, Platts DG, Raffel C, Gabrielian L, Maybauer MO, Barnett A et al: Colour coded microspheres and histological analysis for cerebral mapping: an experimental model. Journal of Neurology Research 2014, 4(1):7-14.

13. Maybauer DM, Maybauer MO, Traber LD, Westphal M, Nakano YY, Enkhbaatar P, Morita N, Herndon DN, Traber DL: Effects of severe smoke inhalation injury and septic shock on global hemodynamics and microvascular blood flow in sheep. Shock 2006, 26(5):489-495.

14. Videtta W, Villarejo F, Cohen M, Domeniconi G, Santa Cruz R, Pinillos O, Rios F, Maskin B: Effects of positive end-expiratory pressure on intracranial pressure and cerebral perfusion pressure. Acta Neurochir Suppl 2002, 81:93-97.

15. Lewis SB, Myburgh JA, Thornton EL, Reilly PL: Cerebral oxygenation monitoring by near-infrared spectroscopy is not clinically useful in patients with severe closed-head injury: a comparison with jugular venous bulb oximetry. Crit Care Med 1996, 24(8):1334-1338.

16. Bellapart J, Cuthbertson K, Dunster K, Diab S, Platts DG, Raffel OC, Gabrielian L, Barnett A, Paratz J, Boots R et al: Cerebral Microcirculation during Experimental Normovolaemic Anemia. Front Neurol, 7:6.

17. Simonova G, Tung JP, Fraser JF, Do HL, Staib A, Chew MS, Dunster KR, Glenister KM, Jackson DE, Fung YL: A comprehensive ovine model of blood transfusion. Vox Sang, 106(2):153-160.

18. Hodgetts VE: The dynamic red cell storage function of the spleen in sheep. III. Relationship to determination of blood volume, total red cell volume, and plasma volume. Aust J Exp Biol Med Sci 1961, 39:187-195.

19. Bellapart J, Dunster KR, Diab S, Platts DG, Raffel C, Gabrielian L, Maybauer MO, Barnett A, Boots RJ, Fraser JF: Intracardiac echocardiography guided transeptal 
catheter injection of microspheres for assessment of cerebral microcirculation in experimental models. Cardiol Res Pract, 2013:595838.

20. Finnie JW, Blumbergs PC, Manavis J, Summersides GE, Davies RA: Evaluation of brain damage resulting from penetrating and non-penetrating captive bolt stunning using lambs. Aust Vet J 2000, 78(11):775-778.

21. Bellapart J, Fraser JF: Transcranial Doppler assessment of cerebral autoregulation. Ultrasound Med Biol 2009, 35(6):883-893.

22. Traber DL: Animal models: the sheep. Crit Care Med 2000, 28(2):591-592.

23. Hodeige D, de Pauw M, Eechaute W, Weyne J, Heyndrickx GR: On the validity of blood flow measurement using colored microspheres. Am J Physiol 1999, 276(4 Pt 2):H1150-1158.

24. Prinzen FW, Bassingthwaighte JB: Blood flow distributions by microsphere deposition methods. Cardiovasc Res 2000, 45(1):13-21.

25. Hakkinen JP, Miller MW, Smith AH, Knight DR: Measurement of organ blood flow with coloured microspheres in the rat. Cardiovasc Res 1995, 29(1):74-79.

26. Itoh T, Satou T, Nishida S, Tsubaki M, Hashimoto S, Ito H: Expression of amyloid precursor protein after rat traumatic brain injury. Neurol Res 2009, 31(1):103109.

27. Stiefel MF, Spiotta A, Gracias VH, Garuffe AM, Guillamondegui O, MaloneyWilensky E, Bloom S, Grady MS, LeRoux PD: Reduced mortality rate in patients with severe traumatic brain injury treated with brain tissue oxygen monitoring. J Neurosurg 2005, 103(5):805-811.

28. Lewis SB, Finnie JW, Blumbergs PC, Scott G, Manavis J, Brown C, Reilly PL, Jones NR, McLean AJ: A head impact model of early axonal injury in the sheep. $J$ Neurotrauma 1996, 13(9):505-514.

29. Sherriff FE, Bridges LR, Sivaloganathan S: Early detection of axonal injury after human head trauma using immunocytochemistry for beta-amyloid precursor protein. Acta Neuropathol 1994, 87(1):55-62.

30. Blumbergs PC, Scott G, Manavis J, Wainwright H, Simpson DA, McLean AJ: Topography of axonal injury as defined by amyloid precursor protein and the sector scoring method in mild and severe closed head injury. J Neurotrauma 1995, 12(4):565-572.

31. Van Den Heuvel C, Blumbergs P, Finnie J, Manavis J, Lewis S, Jones N, Reilly P, Pereira R: Upregulation of amyloid precursor protein and its mRNA in an experimental model of paediatric head injury. J Clin Neurosci 2000, 7(2):140-145.

Tables

Table I: Mean arterial blood pressure (MAP), Intracranial Pressure (ICP) and cerebral perfusion pressure (CPP) values in all subjects at each time point

Table II: SV02 and CO for all subjects

Table III: PH / PC02 and P02 values per subject at each time points

Table IV: Haemoglobin levels (g/dL) for all subjects over time

Table V: Partial Pressure of Tissue Oxygenation $(\mathrm{mmHg})$ for all subjects over time 


\section{Figure legends}

Figure 1:

RMBF for all subjects at all regions of interest over time

Figure 2:

Mean $\mathrm{RMBF}$ at all regions of interest over time

Figure 3:

Line plots of Ratio from baseline for all subjects and all regions of interest The horizontal line at a ratio of 1 indicates no change from baseline

Figure 4:

Mean line plots of Ratio from baseline for all subjects and all regions of interest The horizontal line at a ratio of 1 indicates no change from baseline

Figure 5:

Means and 95\% confidence intervals (vertical lines) from the mixed models. Times where the confidence intervals do not cross the horizontal reference line at 1 indicate a statistically significant change from baseline.

Figure 6: RMBF at extra-cranial regions: the skin, heart, kidney and spleen 